\title{
Micro-computed tomography-based highly automated 3D segmentation of the rat spine for quantitative analysis of metastatic disease
}

\author{
Technical note
}

\author{
Seyed-Parsa Hojjat, M.Eng., Michael R. Hardisty, M.A.Sc., and Cari M. Whyne, Ph.D. \\ Orthopaedic Biomechanics Laboratory, Sunnybrook Health Sciences Centre, Toronto, Ontario, Canada
}

\begin{abstract}
Noninvasive evaluation of metastatic disease in the spine has generally been limited to $2 \mathrm{D}$ qualitative or semiquantitative analysis techniques. This study aims to develop and evaluate a highly automated micro-CT-based quantitative analysis tool that can measure the architectural impact of metastatic involvement in whole vertebrae. Micro-CT analysis of rat whole vertebrae was conducted using a combination of demons deformable registration, level set curvature evolution, and intensity based thresholding techniques along with upsampling and edge enhancement techniques. The algorithm was applied to 6 lumbar vertebrae (L1-3) from $6 \mathrm{rnu} / \mathrm{rnu}$ rats $(3$ healthy rats and 3 with metastatic involvement). Osteolytic metastatic involvement was modeled via MT1 human breast cancer cells. Excellent volumetric concurrency was achieved in comparing the automated micro-CT-based segmentations of the whole vertebrae, trabecular centrums, and individual trabecular networks to manual segmentations $(98.9 \%, 96.1 \%$, and $98.3 \%$, respectively; 6 specimens), and the automated segmentations were achieved in a fraction of the time. The algorithm successfully accounted for discontinuities in the cortical shell caused by vasculature and osteolytic destruction. As such, this work demonstrates the potential of this highly automated segmentation tool to permit rapid precise quantitative structural analysis of the spine with minimum user interaction in the analysis of both healthy and pathological (metastatically involved) vertebrae. Future optimization and the incorporation of lower-resolution imaging parameters may allow automated analysis of clinical CT-based measures in addition to preclinical micro-CT-based analyses of the structural impact and progression of pathological processes in the spine.
\end{abstract}

(DOI: 10.3171/2010.3.SPINE09576)

KEY WoRDs $\quad$ automated segmentation
vertebral metastasis $\bullet \quad$ bony architecture

$\mathrm{M}$ ETASTatic involvement in bone is most frequently seen in the vertebral column. ${ }^{15}$ Skeletal metastases are categorized as osteolytic (bonedestroying), osteoblastic (bone-depositing), or mixed (osteolytic/osteoblastic) lesions. Spinal metastases can result in severe clinical consequences both in terms of morbidity and mortality., ${ }^{5,14}$ Clinical impact is often judged in terms of skeletal-related events (SREs), which include vertebral fracture. ${ }^{4}$ Better measures to assess clinical impact of these lesions prior to fracture would improve patient options and reduce morbidity. As such, radiological measures of metastatic disease in the spine may be used to provide important information related to disease progression as well as evaluation of treatment effects. Robust quantitative evaluation of treatment effects is important both clinically and in preclinical models used to develop and test new and existing therapeutic approaches for spinal metastases.

Noninvasive evaluation of metastatic disease in bone has generally been qualitative or semiquantitative and has historically used 2D analysis techniques. ${ }^{10}$ A number of groups have developed quantitative methods for analyzing bony architecture using CT and micro-CT images, ${ }^{2,3}$ with recent work also focusing on semiautomated quantitative CT-based analysis segmenting and analyzing metastatic involvement specifically in human vertebral bodies (excluding the posterior elements). ${ }^{8,16}$ However, the resolution of conventional CT precludes the analysis of trabecular architecture in the spine, and resolution issues present further challenges in segmentation of the articulations in the posterior elements (facets). Recent work in developing automated segmentation algorithms for trabecular structural analysis in the femur have been limited in their ability to function in the presence of osteolysis and vessels penetrating the cortex. ${ }^{1}$

As such, the objective of this work was to develop and evaluate a highly automated micro-CT-based quantitative analysis tool that is able to measure the impact of metastatic involvement in whole vertebrae. It is hypothesized that highly automated multilevel image analysis applied to 3D micro-CT reconstructions will yield accurate and repeatable segmentation of whole vertebrae, 


\section{S. P. Hojjat, M. R. Hardisty, and C. M. Whyne}

with and without osteolytic defects, in a preclinical rat model. The development of such an automated tool would allow for robust and repeatable measurement of the effects of metastatic disease and treatments on vertebral bone, crucial to evaluating spinal stability.

\section{Methods}

\section{Image Acquisition and Automated Segmentation}

A CT-based tracking tool that had been previously developed for human vertebral body images ${ }^{8}$ was expanded and applied to microimage analysis of rat whole vertebrae (including the posterior elements). The tool aims to automate accurate segmentation of the vertebrae at multiple levels using demons deformable registration followed by level set curvature evolution within the Amira software platform incorporating algorithms from the ITK toolkit (AmiraDEV 3.1, ITK). ${ }^{12,13}$

Micro-CT scans were acquired of rat spinal motion segments $(\mathrm{L} 1-3)(17.5 \times 17.5 \times 17.5 \mu \mathrm{m} /$ voxel, GE Explore Locus, General Electric Co.) using an x-ray source at $90 \mu \mathrm{A}$ and $80 \mathrm{kV}$, with 907 views covering $360^{\circ}$ of rotation. An atlas (consisting of a segmentation and scan) was created of a single L1-3 spinal motion segment (L13) through manual segmentation of one individual specimen.

The automated segmentation begins with registration of the previously obtained atlas scan from a corresponding spinal level to the target vertebra of interest. Prior to the registration, the vertebral bodies of the atlas and fixed target scans are manually aligned to ensure fast convergence of the algorithm. The segmentation procedure is performed in 2 steps. An automated affine registration with 12 degrees of freedom, allowing for shearing, scaling, rotation, and translation is performed to initially register the atlas to the fixed target scan followed by deformable registration. Demons deformable registration is employed using 15 resolution levels with 1000 iterations in each level to achieve a higher degree of accuracy. ${ }^{13}$ Once one resolution level is complete, the deformation field is smoothed using a 3D gaussian filter. The same procedure is performed on the next higher resolution level until all 15 levels are complete.

The obtained deformation field is used to deform the atlas yielding a satisfactory segmentation of the vertebral body of interest; however, this alone does not ensure full inclusion of the posterior elements in the segmentation contour. The attained segmentation is then refined using the level set method to ensure inclusion of high curvature areas such as the posterior elements and to smooth

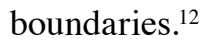

The level set filter is applied in 3 steps to: 1) refine the demons segmentation of the whole vertebrae, 2) move the segmentation boundaries inward to define the cortical shell, and 3) segment the trabecular centrum. Moving the segmentation boundaries inward to be within the cortical shell is an essential intermediate step, as experience has shown that the segmentation curve does not always contract to come within the trabecular centrum without this initial push. ${ }^{8}$
Starting with the output of the demons deformable registration, the parameters are set to cause the curve to flow outward to accurately grasp all the high curvature regions including the posterior elements. To refine the demons segmentation of the entire vertebra the initial speed function is set to cause quick contour contraction through soft tissue (low-intensity values) and slow contour contraction through the bone (high intensity). The level set propagation term and the curvature term are used to smooth out areas of high curvature to prevent the segmentation boundaries from advancing into lytic tumors that have breached the cortical shell. The evolution runs for 60 iterations to ensure the inclusion of the posterior elements in the segmentation. In the second step of level set, the propagation term is increased and the evolution runs for 70 iterations with image intensity values set to a constant derived based on the intensity values of the scan. For the final step, the pixel intensity range is reduced and its center increased to cause high-speed motion through high image intensity (cortical bone) and slow motion through middle and low image intensity (trabecular bone and soft tissue), again increasing the propagation term and run for 50 additional iterations. These assignments help to ensure the segmentation boundary does not move quickly through and hence exclude any blastic tumor tissue connected to the cortical shell when segmenting the trabecular centrum.

Once the trabecular centrum segmentation is obtained, the scan and the segmentation are upsampled by 4 in all directions while preserving the edges of the image using the Lanczos filter (AmiraDev 3.1). This is done to separate the high-intensity voxels from low-intensity voxels as much as possible while limiting the size of the resulting file. Another 2 iterations of the Lanczos filter are applied to the upsampled image to enhance the edges that are blurred due to the upsampling. An intensity-based thresholding is then performed on the output image to segment individual trabeculae. An intensity-based cutoff value $(750 \mathrm{HU})$ was used to identify all higher-intensity voxels as bone and the lower-intensity voxels as not bone (that is, bone marrow or lytic tumor). The output of this step yields segmentation of the individual trabecular network.

\section{Application and Evaluation}

The algorithm was applied to 6 lumbar vertebrae (L-1 to L-3) excised from 3 healthy rnu/rnu rats and 3 rats with metastatic vertebral involvement. Metastatic involvement was modeled via intracardiac injection of MT1 human breast cancer cells, which produces osteolytic vertebral lesions.

After each step of segmentation in each of the 12 specimens (whole vertebrae, trabecular centrum/cortical shell, and individual trabecular network), the algorithm was evaluated using a volumetric concurrency metric. This metric utilizes the volume of the manual segmentation, the volume of the segmentation achieved by the automated algorithm, and the volume of the voxels that were present in both manual and automatic segmentations to compare the output of the algorithm to that of a manual segmentation. For assessment of the trabecular network, 
a volume consisting of 3 adjacent slices was manually refined for each specimen and compared with the volume segmented automatically.

\section{Results}

A volumetric concurrency of $98.89 \% \pm 0.85 \%$ was calculated when automated micro-CT-based segmentations of the whole vertebrae were compared with manual segmentations (6 specimens). The manual segmentations took an average of 12 hours per specimen while the automated segmentation took on average 40 minutes to complete with minimal user interaction. Using level set curvature evolution, the algorithm was able to account for discontinuities in the cortical shell. This allows representative volumes to be generated despite vessels and nerves entering the vertebral body and enables segmentation of vertebrae in which lytic tumor has destroyed portions of the cortical shell (Fig. 1).

For the trabecular centrum, a volumetric concurrency of $96.08 \% \pm 3.03 \%$ was calculated when the automated micro-CT-based segmentations were compared with the manual segmentations (6 specimens). Following the initial segmentation of the whole vertebrae, the manual segmentations of the trabecular centrum took an average of 6 hours per specimen while the automated segmentation took on average 15 minutes to complete. Volume of the cortical shell was calculated through subtraction of the trabecular segmentation from the whole body segmentation and demonstrated similar volumetric concurrency when comparing the automated versus manual analyses $(96.12 \% \pm 3.17 \%)$.

A volumetric concurrency of $98.25 \% \pm 0.62 \%$ was calculated when the automated micro-CT-based segmentations of the individual trabeculae were compared with the manual segmentations (6 specimens). The manual method using intensity-based thresholding followed by manual refinement took on average 15 minutes (for the 3 -slice volume) while the automated algorithm took on average 1 minute to complete. Upsampling and edge enhancement were necessary to yield accurate segmentation of the individual trabecular network.

\section{Discussion}

This work is a development of an accurate highly au- tomated segmentation tool that enables precise quantitative structural analysis of the spine with minimum user interaction. Importantly, this tool was successful in the analysis of both healthy and pathologic (metastatically involved) vertebrae. The algorithm employs atlas-based deformable registration that is significantly faster than manual methods. The execution time of the algorithm is dependent on size of the $\mu \mathrm{CT}$ image, meaning that in applications where less accuracy is required, the speed of the algorithm could be increased by downsampling or downsizing the scanning parameters (lower resolution). Automated vertebral segmentation in reasonable analysis times will allow for widespread application of 3D spinal analysis. Automated methods remove inter-/intra-user variability and minimal user intervention reduces cost, facilitating the application of 3D quantitative measures. Reasonable analysis times and reduced costs also motivate the potential for quantitative analysis in larger research studies, increasing their potential power.

Accurate segmentation is necessary for evaluating bony architecture. Evaluating bone quality, in contrast to bone density alone, is essential in representing the structural integrity of the spine. ${ }^{11}$ Architectural measurements allow for robust analyses to evaluate disease state, progression, and response to new or existing local or systemic therapies. The level set curvature evolution method is of major importance in achieving accurate segmentation in the metastatic spine as it prevents the segmentation from collapsing when the cortical shell is severely damaged by the tumor. The metastatically involved vertebrae analyzed varied in tumor burden, representing differing levels of metastatic involvement, from a large defect breaching the cortical shell to a smaller contained lesion within the trabecular centrum. The ability of the tool to function in segmenting structurally compromised vertebrae with defects due to osteolysis at multiple stages is essential to evaluating metastatic disease progression and the ability of treatments to structurally improve vertebral stability. Accurate assessment of vertebral stability and fracture risk are of the utmost importance in clinical decisions related to surgical intervention.

Recent studies have demonstrated the feasibility of CT-based 3D automated segmentation methods. De Nunzio et $a .^{6}$ successfully used a region-growing approach to segment the anatomy of the lung. A combination of thresholding and ellipse fitting has also been used to au-

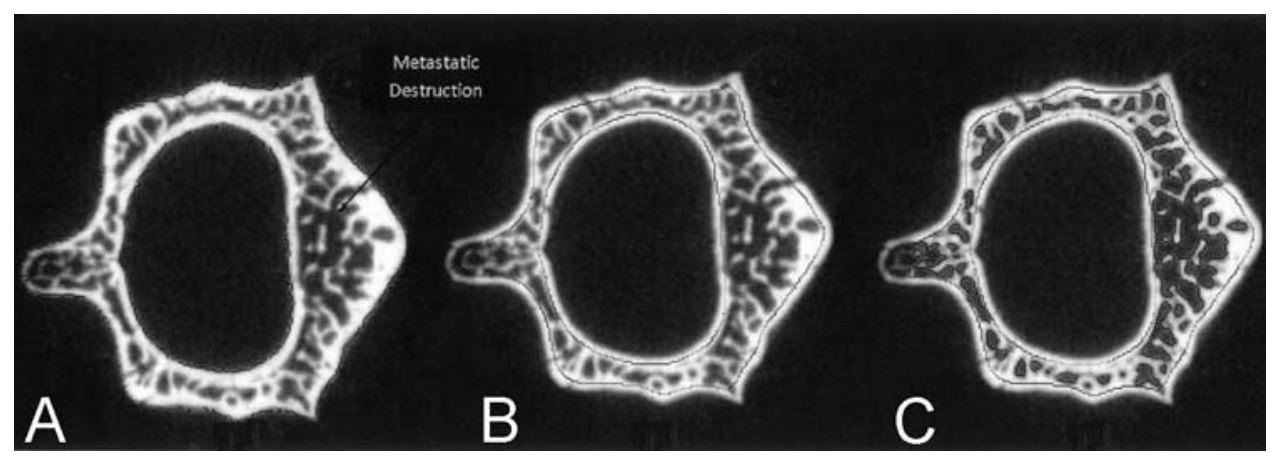

Fig. 1. Representative micro-CT scans showing segmentation of the whole vertebra (A), segmentation of the trabecular centrum (B), and segmentation of the trabeculae (C). 


\section{S. P. Hojjat, M. R. Hardisty, and C. M. Whyne}

tomatically segment the proximal femur; however, this method proved to be limited in its ability to function in the presence of discontinuities (osteolysis and vessels penetrating the cortex).${ }^{15}$ In contrast, the level set method can overcome discontinuities in bony segmentation. The developed algorithm was able to use curve expansion to segment whole rat vertebrae and contraction to segment the trabecular centrum while maintaining an accurate shape by flipping the sigmoid function. The output in both cases was a smooth segmentation curve that was still able to grasp high-curvature area such as the posterior elements.

The resolution level of the images yielded clear delineation between the bony articulations at the facets, thus additional image processing was not required to ensure accurate segmentation at these sites. These segmentations, with accurate definitions of facet geometry and osteolytic defects, provide a robust platform for further mathematical modeling, such as finite element analysis. Finite element analysis represents a powerful tool to quantify mechanical stability of the spine in healthy and pathological states. The ability of the tool to yield multiple segmentations (whole bone to trabecular network) further provides a basis for the appreciation of multiscale differences in finite element analysis of vertebral bone?

In this work, we have presented an accurate, fast, and highly automated tool for segmentation of rat spines into whole vertebrae, cortical shells/trabecular centrums, and their individual trabecular networks. The algorithm has employed a combination of demons deformable registration, level set curvature evolution, and intensity-based thresholding techniques along with upsampling and edge enhancement to ensure accurate 3D segmentation of the rat spine. The automated segmentations have been evaluated in comparison with manual segmentations yielding a volumetric concurrency of approximately $95 \%$ while drastically reducing the execution time of the manual segmentations. While this tool has been developed for preclinical analysis of whole rat vertebrae, the methods employed can be translated to improve automated techniques to segment full human vertebrae (including the posterior elements) with extensive osteolytic and/or osteoblastic disease. Future extension of this work may be used to optimize and incorporate lower resolution imaging parameters to better quantify and automate clinical CT-based measures of the structural impact and progression of pathological processes, such as metastatic disease, and associated treatments on spinal stability.

\section{Disclosure}

The authors report no conflict of interest concerning the materials and methods used in this study or the findings specified in this paper.

Supported by Canadian Institute of Health Research (CIHR) grant \# 724180902.

Author contributions to the study and manuscript preparation include the following. Conception and design: all authors. Acquisition of data: Hojjat, Hardisty. Analysis and interpretation of data: all authors. Drafting the article: Whyne, Hojjat. Critically revising the article: Whyne, Hojjat. Reviewed final version of the manuscript and approved it for submission: Whyne. Administrative/ technical/material support: Whyne. Study supervision: Whyne.

\section{Acknowledgments}

The authors would like to thank Dr. Margarete Akens for her work on the preclinical model.

\section{References}

1. Baum T, Carballido-Gamio J, Huber MB, Müller D, Monetti $\mathrm{R}$, Räth $\mathrm{C}$, et al: Automated $3 \mathrm{D}$ trabecular bone structure analysis of the proximal femur-prediction of biomechanical strength by CT and DXA. Osteoporos Int:October 27, 2009 [Epub ahead of print]

2. Boyd SK, Müller R: Smooth surface meshing for automated finite element model generation from 3D image data. J Biomech 39:1287-1295, 2006

3. Buie HR, Campbell GM, Klinck RJ, MacNeil JA, Boyd SK: Automatic segmentation of cortical and trabecular compartments based on a dual threshold technique for in vivo microCT bone analysis. Bone 41:505-515, 2007

4. Coleman RE, Rubens RD: The clinical course of bone metastases from breast cancer. Br J Cancer 55:61-66, 1987

5. Constans JP, de Divitiis E, Donzelli R, Spaziante R, Meder JF, Haye C: Spinal metastases with neurological manifestations. Review of 600 cases. J Neurosurg 59:111-118, 1983

6. De Nunzio G, Tommasi E, Agrusti A, Cataldo R, De Mitri I, Favetta M, et al: Automatic lung segmentation in CT Images with accurate handling of the Hilar region. J Digit Imaging:October 14, 2009 [Epub ahead of print]

7. Eswaran SK, Fields AJ, Nagarathnam P, Keaveny TM: Multiscale modeling of the human vertebral body: comparison of micro-CT based high-resolution and continuum-level models. Pac Symp Biocomput:293-303, 2009

8. Hardisty M, Gordon L, Hardisty M, Agarwal P, Skrinskas T, Whyne C: Quantitative characterization of metastatic disease in the spine. Part I. Semi-automated segmentation of the metastatic spine using atlas-based deformable registration and the level set method. Med Phys 34:3127-3134, 2007

9. Houston SJ, Rubens RD: The systemic treatment of bone metastases. Clin Orthop Relat Res 312:95-104, 1995

10. Jacobs WB, Perrin RG: Evaluation and treatment of spinal metastases: an overview. Neurosurg Focus 11(6):e10, 2001

11. Odgaard A: Three-dimensional methods for quantification of cancellous bone architecture. Bone 20:315-328, 1997

12. Osher S, Sethian JA: Fronts propagating with curvature-dependent speed: algorithms based on Hamilton-Jacobi formulations. J Comput Phys 79:12-49, 1988

13. Thirion JP: Image matching as a diffusion process: an analogy with Maxwell's demons. Med Image Anal 2:243-260, 1998

14. Toma S, Venturino A, Sogno G, Formica C, Bignotti B, Bonassi S, et al: Metastatic bone tumors. Nonsurgical treatment. Outcome and survival. Clin Orthop Relat Res 295:246-251, 1993

15. Wong DA, Fornasier VL, MacNab I: Spinal metastases: the obvious, the occult, and the impostors. Spine 15:1-4, 1990

16. Whyne CM, Hardisty M, Wu F, Skrinskas T, Clemons M, Gordon L, et al: Quantitative characterization of metastatic disease in the spine. Part II. Histogram-based analyses. Med Phys 34:3279-3285, 2007

Manuscript submitted June 30, 2009.

Accepted March 30, 2010

Address correspondence to: Cari M. Whyne, Ph.D., Orthopaedic Biomechanics Laboratory, Sunnybrook Health Sciences Centre, Room UB19, 2075 Bayview Avenue, Toronto, Ontario, Canada, M4N 3M5. email: cari.whyne@sunnybrook.ca. 